\title{
Inclusão do resíduo de polpação da celulose na produção de painéis aglomerados de média densidade
}

\author{
Inclusion of cellulose pulping waste for production of medium density \\ particleboards
}

\author{
Láysa Maria Ferreira Andrade ${ }^{1}$, Mário Vanoli Scatolino', \\ Douglas Lamounier Faria ${ }^{1}$, Antônia Amanda César ${ }^{1}$, Lourival Marin Mendes ${ }^{1}$ \\ e José Benedito Guimarães Junior ${ }^{1}$
}

\begin{abstract}
RESUMO
O processo de produção de celulose gera diversos resíduos, dentre eles o material resultante da depuração como o UKP (Unbleach Kraft Pulp). O emprego desses resíduos na fabricação de produtos pode agregar valor e solucionar problemas ambientais, além de contribuir para o desenvolvimento de novas tecnologias. O objetivo deste trabalho foi avaliar o potencial de utilização do resíduo UKP, na produção de painéis MDP. Para a produção dos painéis foram utilizados a madeira de Eucalyptus sp. e o resíduo UKP. Os tratamentos consistiram em diferentes porcentagens de partículas do resíduo em mistura com partículas de Eucalyptus $s p$. na fração do miolo ( $25 \%, 50 \%, 75 \%$ e $100 \%$ de UKP), ainda foram produzidos painéis com $100 \%$ das partículas de eucalipto (testemunha). Os painéis foram confeccionados com densidade nominal de $0,70 \mathrm{~g} / \mathrm{cm}^{3}$; relação face/miolo/face de 20:60:20 e 12\% de adesivo uréia-formaldeído tanto para as camadas das faces quanto do miolo. O ciclo de prensagem consistiu de uma pressão específica de $3,92 \mathrm{MPa}$, temperatura de $160^{\circ} \mathrm{C}$ e tempo de 8 minutos. Os seguintes testes foram realizados: absorção de água após 2 e $24 \mathrm{~h}$ de imersão, inchamento em espessura após 2 e 24 h de imersão, densidade aparente, taxa de não retorno em espessura, umidade, tração perpendicular e flexão estática. Os valores para as propriedades físicas de absorção de água e inchamento em espessura após 24 h variaram de 53 a $130 \%$ e de 8 a $50 \%$, respectivamente, de acordo com o aumento da quantidade de resíduo na composição dos painéis. De modo semelhante, houve um decréscimo nos valores de MOE (de 2264,82 para 543,72 MPa) e MOR (de 19,66 para 3,46 Mpa). Recomenda-se a utilização de até 35\% de substituição de partículas de madeira por partículas do resíduo UKP para produção dos painéis MDP, permitindo, assim, uma destinação do rejeito na geração de novos produtos, mantendo as propriedades físicas e mecânicas dentro dos valores exigidos pelas normas.
\end{abstract}

Palavras-chave: Rejeito; polpação; indústria de celulose.

\begin{abstract}
The pulp production process generates several residues, among them, the material resulting from the purification known as UKP (Unbleached Kraft Pulp). The use of waste in the manufacture of products can add value and solve environmental problems, as well as contributing to the development of new technologies. The objective of this work was to evaluate the potential of using UKP (Unbleached Kraft Pulp) and wood of Eucalyptus sp. for the production of MDP panels. The treatments consisted of different percentages of particles of the residue in mixture with particles of Eucalyptus sp. (25\%, 50\%, $75 \%$ and $100 \%$ of UKP), and $100 \%$ of the eucalyptus particles (control). The panels were made with nominal density of $0.70 \mathrm{~g} / \mathrm{cm}^{3}$; face / core / face ratio of 20:60:20; $12 \%$ urea-formaldehyde adhesive for the faces and core. The pressing cycle consisted of a dose of $3.92 \mathrm{MPa}$, a temperature of $160^{\circ} \mathrm{C}$ and a time of 8 minutes. The following tests were performed: water absorption after 2 and $24 \mathrm{~h}$ of immersion, swelling in thickness after 2 and $24 \mathrm{~h}$ of immersion, apparent density, and non-return rate in thickness, humidity, perpendicular traction and static bending. The values for the physical properties of water absorption and thickness swelling after $24 \mathrm{~h}$ ranged from 53 to $130 \%$ and from 8 to $50 \%$, respectively, according to the increase of the waste amount in the panels composition. Similarly, there was a decrease in MOE values (from 2264.82 to $543.72 \mathrm{Mpa}$ ) and MOR (from 19.66 to $3.46 \mathrm{Mpa}$ ). It is recommend to use $35 \%$ replacement of wood particles by UKP waste particles for the production of MDP panels, thus allowing a waste disposal in the generation of new products, while maintaining the physical and mechanical properties within the required values standards.
\end{abstract}

Keywords: Waste; pulping; pulp industry. 


\section{INTRODUÇÃO}

A busca pela redução dos descartes industriais e o aumento do aproveitamento das matérias primas, vem mostrando oportunidades de recuperação dos resíduos, que podem ter uma utilização mais nobre, como a fabricação de novos produtos. O processo de produção da celulose é responsável por parte dos resíduos industriais produzidos no Brasil. Os principais resíduos gerados neste processo correspondem ao lodo biológico, produzidos no tratamento de fluentes, as cascas de madeira, a lama de cal e outros materiais alcalinos da planta de caustificação (dregs e gifts), além dos rejeitos da depuração da polpa marrom e da polpa branqueada. Segundo Neuberger (2008), o teor de rejeitos está relacionado às características da matéria-prima madeira e dos parâmetros do processo de polpação, principalmente tempo e temperatura de impregnação, fator H e carga alcalina.

Os rejeitos da depuração denominados "Unbleach Kraft Pulp" (UKP) e "Bleach Kraft Pulp" (BKP), considerados rejeitos da produção, são ricos em fibras e, na maioria das vezes, descartados em aterros sanitários após tratamento (GAMA et al., 2010). Além da poluição ambiental causada pelo descarte deste material, o gasto da empresa para tratar o resíduo antes de descartar deve ser levado em consideração. A utilização deste resíduo para produção de qualquer produto que agregue valor é interessante, tanto para o meio ambiente, quanto para a indústria de celulose (CÉSAR, 2015). Uma alternativa para o emprego do resíduo seria na produção de painéis aglomerados.

A literatura relata vários estudos com a utilização de resíduos agrícolas ou industriais na produção de painéis aglomerados como bagaço de cana (MENDES et al., 2012), casca de arroz (MELO et al., 2009), sabugo de milho (SCATOLINO et al., 2015; 2013; SEKALUVU et al., 2014), casca de café (MENDES et al., 2010), casca de amendoim (GULER; BUYUKSARI, 2011), palha de milho (SILVA et al., 2015), podas de videira (Vitis vinifera L.) (YENIOCAK et al., 2014) e casca de mamona (SILVA et al., 2016).

Painéis aglomerados convencionais são produtos gerados com partículas do tipo sliver de qualquer material lignocelulósico, onde a união destes é efetuada pela utilização de adesivo sintético que são consolidados sob pressão e temperatura (SORATTO et al., 2013). O MDP (Medium Density Particleboard) é considerado uma evolução no processo de fabricação dos aglomerados convencionais, no qual as partículas de menores dimensões ficam nas camadas superficiais e as maiores no miolo ou camada interna (MESQUITA et al., 2015).

De acordo com o relatório anual da Indústria Brasileira de Árvores (IBÁ, 2017), o segmento de produção de painéis de madeira e pisos laminados, corresponde a $6 \%$ da distribuição das áreas de árvores plantadas no Brasil, possui 18 unidades produtoras de painéis de madeira e ocupa o $8^{\circ}$ lugar no ranking mundial dos maiores produtores. Somente no caso do MDP, a produção em 2016 foi de 3,0 milhões de metros cúbicos. Conforme destaca Barros Filho (2009), parcela preponderante da matéria prima para a fabricação dos painéis particulados ainda é proveniente de árvores inteiras, oriundas de áreas de florestas plantadas.

Neste contexto, diversos estudos têm empregado resíduos lignocelulósicos oriundos do setor moveleiro, de processos agrícolas ou industriais para produção de painéis reconstituídos de madeira e compósitos. Pierre et al. (2014) produziram MDP's com resíduos industriais de paletes e serragem. Iwakiri et al. (2000) avaliaram a viabilidade de utilização da madeira de E. maculata, E. grandis e E. tereticornis, na forma de resíduos de processamento em serrarias, para produção de painéis de madeira aglomerada. Da mesma forma, Pedrazzi et al. (2006) avaliaram a qualidade de painéis aglomerados fabricados com partículas e serragem de madeira de E. saligna, colados com adesivo à base de uréia-formaldeído. O resíduo UKP já foi utilizado em estudos (MOHR et al., 2005; TONOLI et al., 2013), nos quais foram avaliadas as propriedades mecânicas de compósitos reforçados com o mesmo. Os resultados encontrados permitiram observar uma boa viabilidade de utilização do resíduo. Essas pesquisas podem favorecer o melhor aproveitamento da matéria-prima, proporcionando maior valor agregado ao produto e empregando testes para verificar a qualidade e propriedades de resistência desses produtos. Dessa forma, o objetivo deste estudo foi avaliar o efeito da utilização de diferentes proporções do resíduo UKP sobre as propriedades físicas e mecânicas de painéis MDP.

\section{MATERIAL E MÉTODOS}

O material utilizado foi o resíduo UKP proveniente da fábrica da Cenibra, localizada em Belo Oriente-MG. A espécie utilizada pela empresa é um híbrido entre Eucalyptus urophylla e Eucalyptus grandis com 7 anos de idade. O resíduo (Figura 1) foi recebido na Unidade Experimental em Painéis de 


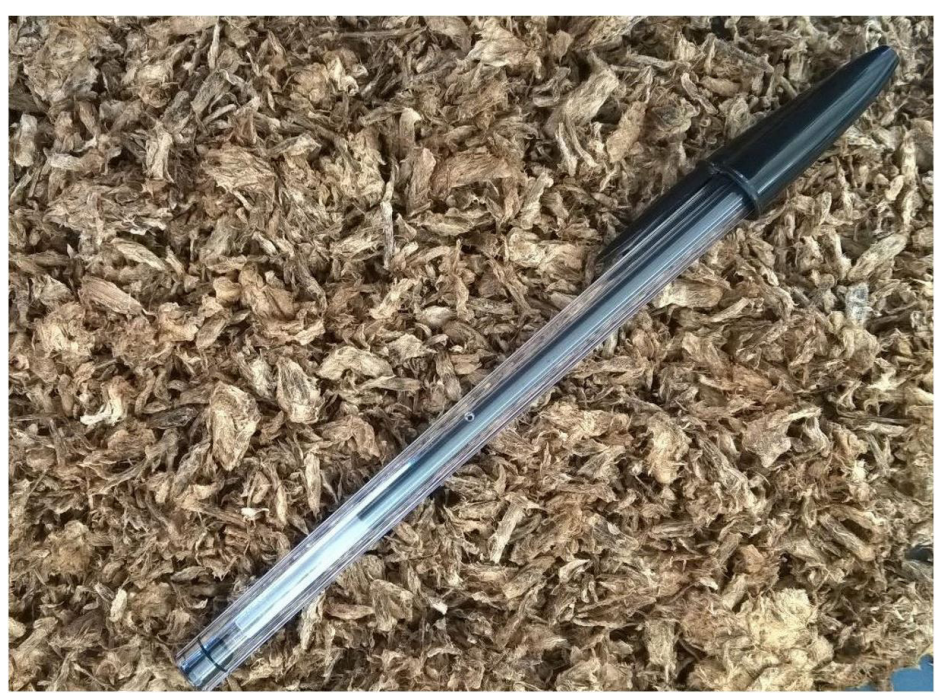

Figura 1. Resíduo fibroso da depuração pós-cozimento na indústria de celulose (UKP) ao natural após secagem ao ar livre.

Figure 1. Fiber residue from the post-firing treatment in the pulp industry (UKP) to the natural after drying.

Madeira (UEPAM/UFLA), e espalhado sobre lonas para que fosse seco ao ar livre, posteriormente foi acondicionado em uma caixa d'água com tampa, até a sua utilização.

Além do resíduo, foram utilizadas árvores de Eucalyptus sp., com idade de 15 anos, idade aproximada do plantio experimental disponível no campus da Universidade Federal de Lavras. Após a coleta, as árvores foram serradas em toretes menores e discos foram removidos para determinação da densidade básica de acordo com a norma ABNT (2003). A densidade do resíduo foi determinada pelo mesmo princípio, porém, o volume saturado foi obtido utilizando-se uma proveta graduada, a qual permitiu a visualização do volume de água deslocado. A madeira de Eucalyptus sp. foi processada em moinho martelo para geração de partículas do tipo sliver.

As partículas de madeira e de resíduo foram secas até 3\% de umidade em estufa de circulação de ar forçada e classificadas para a retirada de finos com uma peneira de abertura 0,6 mm. Posteriormente foram coletadas amostras das partículas para que fossem determinadas suas dimensões. O comprimento das partículas de eucalipto foi obtido por meio de microscopia óptica e apresentou média aproximada de 1,72 mm, tanto as utilizadas nas faces, quanto as destinadas ao miolo. As partículas do resíduo, como eram mais "grosseiras", foram medidas com um paquímetro, tendo comprimento médio de $6,14 \mathrm{~mm}$. O adesivo utilizado foi a uréia-formaldeído, sendo adicionado na massa do painel na porcentagem de $12 \%$ (base massa seca) tanto nas faces quanto no miolo, com teor de sólidos de 55\%, pH de 7,53, viscosidade de 579 cP. Por se tratar de um resíduo cuja aplicação em painéis ainda é desconhecida, optou-se por manter a porcentagem de adesivo em 12\% também para o miolo. $\mathrm{O}$ adesivo foi misturado às partículas de forma manual, separadamente nas faces e miolos, até que todo o material lignocelulósico, seja ele resíduo ou madeira ficasse impregnado com o adesivo. As partículas foram distribuídas no colchão em três camadas nas proporções de 20, 60 e 20\% em relação à massa total do painel para face, miolo e face, respectivamente.

Na Tabela 1 encontram-se os tratamentos realizados. Utilizou-se cinco diferentes proporções do resíduo da depuração misturado à madeira de Eucalyptus sp., porém o resíduo foi adicionado apenas no miolo dos painéis.

Tabela 1. Plano experimental.

Table 1. Experimental plan.

\begin{tabular}{cccc}
\hline \multirow{2}{*}{ Tratamento } & Composição & Eucalipto & UKP \\
\cline { 3 - 4 } & $100 E$ & ---100 & 0 \\
2 & $75 E / 25 U K P$ & 75 & 25 \\
3 & $50 E / 50$ UKP & 50 & 50 \\
4 & $25 E / 75$ UKP & 25 & 75 \\
5 & 100 UKP & 0 & 100 \\
\hline
\end{tabular}


Para a formação do colchão foi utilizada uma caixa formadora com dimensões de 300 x $300 \mathrm{~mm}$. O colchão foi pré-prensado até 0,4 MPa em uma prensa manual. O processo de prensagem a quente foi realizado em uma prensa hidráulica automática com controle de temperatura e pressão, sendo que as variáveis do ciclo foram: temperatura de $160{ }^{\circ} \mathrm{C}$, pressão de 3,92 $\mathrm{MPa}$, por um período de 8 minutos. Todos os painéis foram produzidos com densidade nominal de $0,700 \mathrm{~g} / \mathrm{cm}^{3}$ e espessura de $15 \mathrm{~mm}$. Para cada tratamento, foram produzidos 3 painéis.

Retiraram-se 4 corpos de prova por painel, para avaliação das propriedades de módulo de elasticidade (MOE) e módulo de ruptura (MOR) à flexão estática, que tiveram como base a norma DIN (Deutsches Institut fur Normung) 52362 (DIN, 1982); para o teste de tração perpendicular (TP) foram retirados 6 corpos de prova por painel; 2 corpos de prova por painel para absorção de água (AA) e inchamento em espessura (IE) após 2 e $24 \mathrm{~h}$ de imersão. Tais ensaios se basearam na norma ASTM (American Society for Testing and Materials) - D1037 (ASTM, 2006). Os mesmos corpos de prova utilizados para o teste de inchamento em espessura foram utilizados logo após para a medição da taxa de não retorno em espessura (TNRE) (Eq.1).

$$
T N R E=\frac{E f-E c}{E f} \times 100
$$

onde TNRE é a taxa de não retorno em espessura (\%); Efé a espessura do painel (mm) após pronto e armazenado em ambiente climatizado e Ec é a espessura do painel $(\mathrm{mm})$ após o teste de inchamento em espessura e posterior armazenamento em ambiente climatizado.

As amostras foram climatizadas à temperatura de $20 \pm 3{ }^{\circ} \mathrm{C}$ e umidade relativa de $65 \pm 5 \%$. Posteriormente, alguns corpos de prova foram levados para uma estufa com temperatura de $103 \pm 2{ }^{\circ} \mathrm{C}$, o que permitiu o registro da massa seca de cada corpo de prova e, por consequência, proporcionou a determinação da umidade das amostras.

O delineamento utilizado foi o inteiramente casualizado, com a realização da análise de variância e quando significativa, a análise de regressão linear ou quadrática, ambos a 5\% de significância.

\section{RESULTADOS E DISCUSSÃO}

A densidade aparente dos painéis, obtida pela norma EN 323 (European Committee for Standardization) (EN, 1993), variou de 0,622 a $0,648 \mathrm{~g} / \mathrm{cm}^{3}$, porém não houve diferença significativa entre os tratamentos (Tabela 2).

Tabela 2.Densidade aparente e umidade do painel.

Table 2.Apparent density and humidity of the panel.

\begin{tabular}{cccc} 
Tratamento & $\begin{array}{c}\text { UKP } \\
(\%)\end{array}$ & $\begin{array}{c}\text { Densidade aparente } \\
\left(\mathbf{g} / \mathbf{c m}^{\mathbf{3}}\right)\end{array}$ & $\begin{array}{c}\text { Umidade } \\
(\%)\end{array}$ \\
\hline 1 & 0 & $0,648 \pm 0,030^{*}$ & $8,21 \pm 0,13$ \\
2 & 25 & $0,622 \pm 0,016$ & $8,05 \pm 0,07$ \\
3 & 50 & $0,624 \pm 0,010$ & $7,53 \pm 0,28$ \\
4 & 75 & $0,639 \pm 0,036$ & $7,37 \pm 0,83$ \\
5 & 100 & $0,626 \pm 0,024$ & $7,72 \pm 0,16$ \\
\hline
\end{tabular}

*Desvio padrão

Os painéis podem ser considerados como de média densidade aparente, de acordo com a classificação da ABNT (2006) que estabelece intervalo de 0,55 a 0,75 g/ $\mathrm{cm}^{3}$. Observa-se, contudo, que os valores de densidade aparente foram inferiores aos valores pré-estabelecidos de $0,70 \mathrm{~g} / \mathrm{cm}^{3}$. Isso pode ser atribuído à especificidade das condições laboratoriais em relação ao processo industrial, com perdas de materiais durante o manuseio das partículas nas etapas de aplicação de adesivo, formação do colchão e prensagem dos painéis.

A umidade variou de 7,37 a 8,21\% e não houve diferença estatística entre os tratamentos. A norma brasileira NBR 14810 ABNT (2006) estipula valores de umidade entre 5 e 11\%, sendo assim, todos os tratamentos se enquadram na norma citada.

A Figura 2 apresenta a relação entre a razão de compactação e a porcentagem de resíduo utilizado. Observa-se que o aumento da inserção de fibra ocasiona aumento linear da razão de compactação, sendo que a cada $1 \%$ de inserção de fibra no painel ocorreu um aumento na razão de compactação 


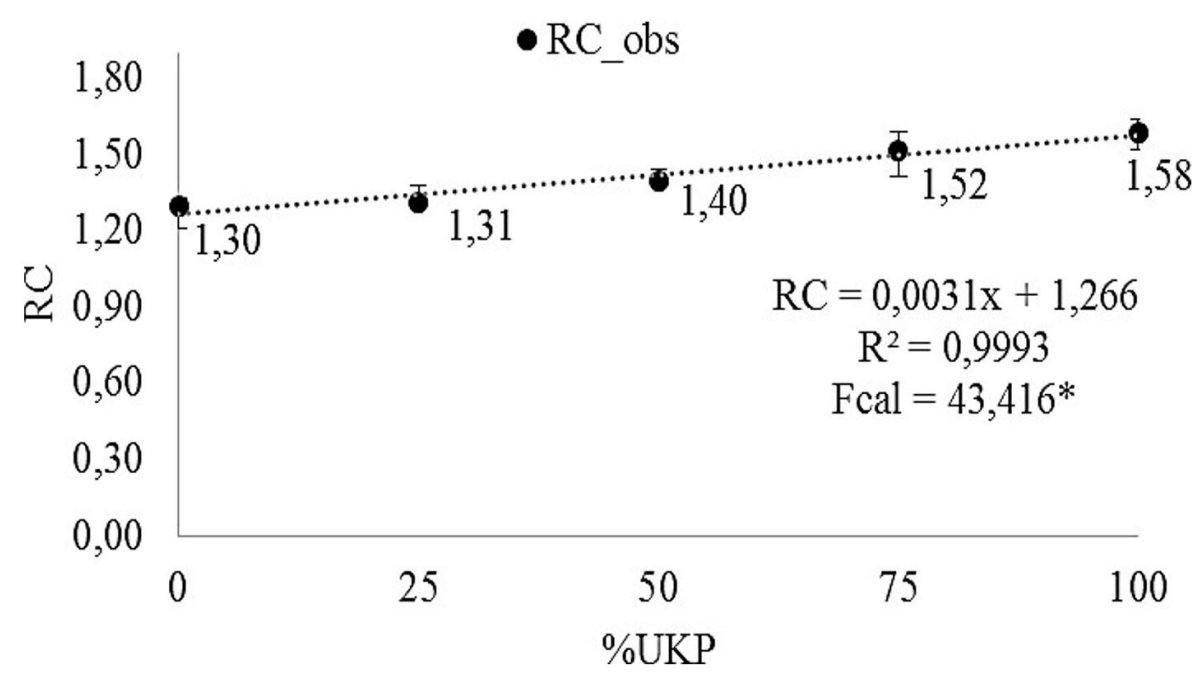

Figura 2. Razão de compactação dos painéis MDP, onde $\left(^{*}\right)$ - significativo ao nível de $5 \%$ de significância.

Figure 2. Compression ratio of the MDP panels, where $\left(^{*}\right)$ - significant at the $5 \%$ level of significance.

na ordem aproximadamente 0,0031 . Tal fato se deve à menor densidade do resíduo $\left(0,325 \mathrm{~g} / \mathrm{cm}^{3}\right)$ quando comparado à densidade do eucalipto $\left(0,586 \mathrm{~g} / \mathrm{cm}^{3}\right)$.

Maloney (1993) relata que a faixa ideal de razão de compactação deve ser entre 1,3 e 1,6. Sendo assim, todos os tratamentos se encontram dentro da faixa. Essa propriedade é um parâmetro de grande importância na resistência mecânica de painéis particulados, uma vez que painéis com maior razão de compactação provavelmente ocasionarão propriedades mecânicas superiores e geralmente menor absorção de água e inchamento em espessura devido a maior dificuldade de penetração de água. No entanto, quando se utiliza resíduos de baixa densidade na formação do painel, esse quadro pode se inverter devido à maior quantidade de partículas por volume. Em casos assim, os painéis podem apresentar menor resistência mecânica e maior absorção de água e inchamento em espessura.

O modelo de regressão ajustado em função do aumento da porcentagem de resíduo UKP para os valores de absorção de água 2 e 24 horas de imersão em água estão apresentados na Figura 3.

O tratamento utilizando $100 \%$ de UKP no miolo não foi resistente a água, não sendo possível à medição das propriedades físicas deste tratamento (Figura 4). Tal fato é um indicativo de que a produção de um painel com 100\% de resíduo UKP no miolo não é uma alternativa viável.

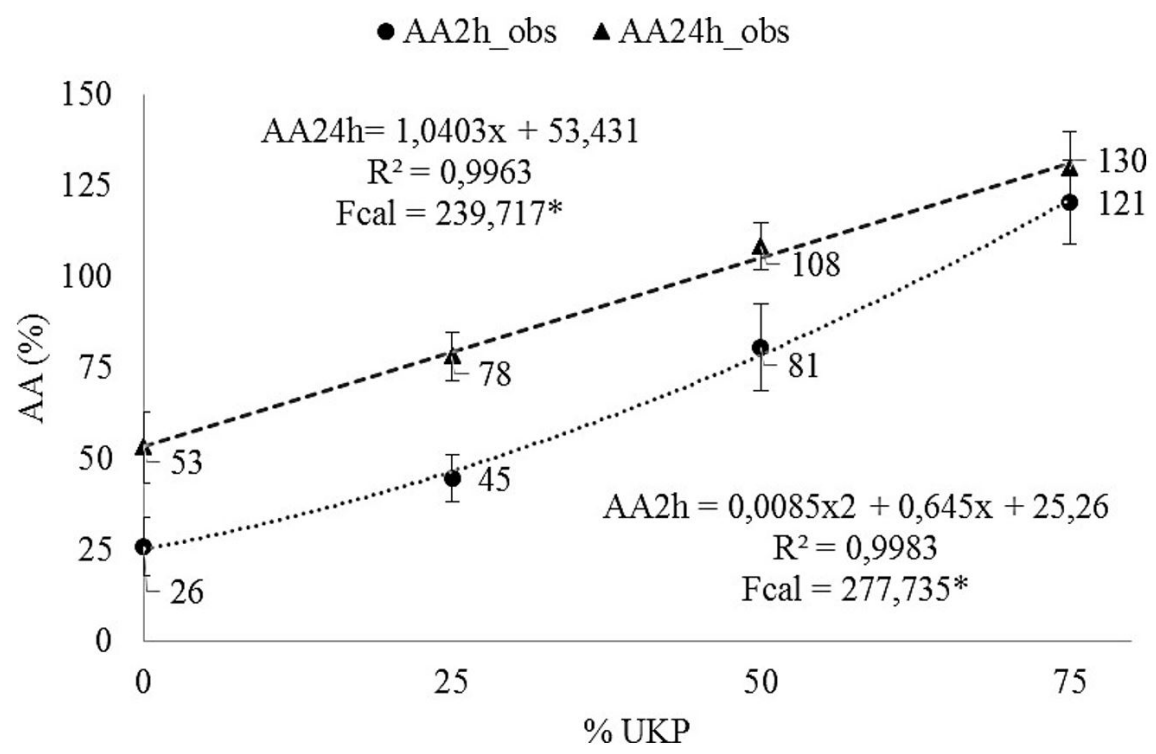

Figura 3. Absorção de água após 2 e 24 horas de imersão dos painéis MDP, onde (*) - significativo ao nível de $5 \%$ de significância.

Figure 3. Water absorption after 2 and 24 hours immersion of the MDP panels, where $(*)-$ significant at the $5 \%$ level of significance. 


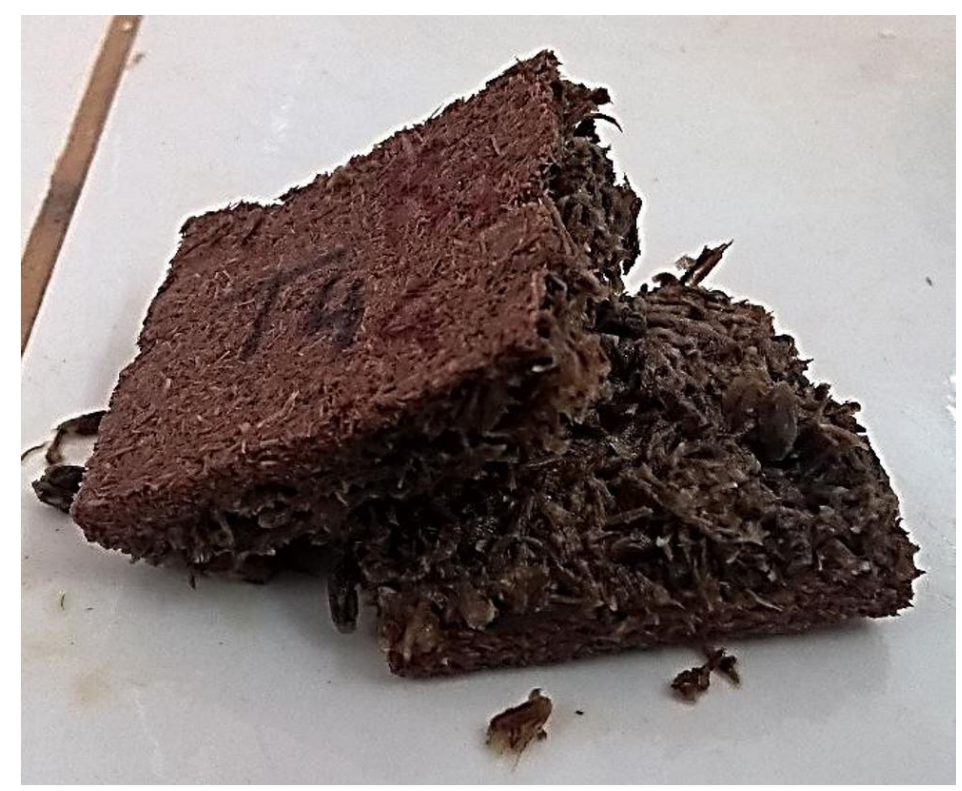

Figura 4. Corpo de prova do tratamento utilizando $100 \%$ de resíduo após 2 horas de imersão.

Figure 4. Sample of the treatment using $100 \%$ waste after 2 hours immersion.

Silva et al. (2016) avaliando a associação da madeira de pinus na com casca de mamona na produção de painéis aglomerados obtiveram valores médios de AA2h e AA24h de 77,60\% e $117,40 \%$, respectivamente, para os painéis compostos apenas por partículas de pinus. De forma similar, Silva et al. (2015) promoveram a associação da madeira de eucalipto com diferentes teores de palha de milho na produção de painéis MDP e encontraram valores médios de AA2h e AA24h de 82 e $91 \%$, respectivamente, para os painéis produzidos unicamente com as partículas de madeira. Neste sentido, os painéis produzidos apresentaram valores médios de AA2h e AA24h dentro dos encontrados na literatura.

A relação entre o IE2h e IE24h e a porcentagem de resíduo UKP utilizado foi uma regressão linear e quadrática significativa para ambas as propriedades (Figura 5), sendo observado aumento dos valores médios com o aumento da quantidade de resíduo utilizado.

Scatolino et al. (2013), avaliando a incorporação de sabugo de milho em painéis de Pinus oocarpa e Silva et al. (2015), verificando a incorporação de palha de milho em painéis de eucalipto observaram os valores de IE $24 \mathrm{~h}$ de 37 e $23 \%$, respectivamente para os painéis produzidos unicamente com partículas de madeira. Tais valores se assemelham ao IE $24 \mathrm{~h}$ encontrado para os painéis em que houve a incorporação de 50\% de resíduo ao miolo (IE $24 \mathrm{~h}$ de $25 \%$ ).

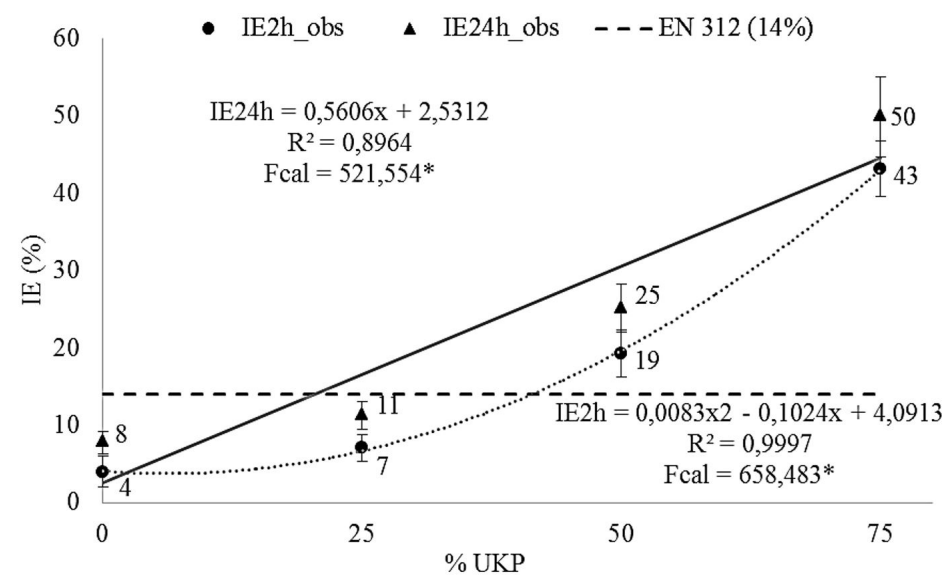

Figura 5. Inchamento em espessura após 2 e 24 horas de imersão dos painéis MDP, onde (*) - significativo ao nível de $5 \%$ de significância.

Figure 5. Swelling in thickness after 2 and 24 hours of immersion of MDP panels, where $\left(^{*}\right)$ - significant at the $5 \%$ level of significance. 
Andrade et al. - Inclusão do resíduo de polpação da celulose na produção de painéis aglomerados de média densidade

A norma EN 312 (EN, 2003) estabelece valor máximo de IE24h de 14\% para painéis aglomerados com adesivo uréia-formaldeído, sendo assim, os painéis produzidos com $25 \%$ de resíduo apresentaram inchamento inferior ao valor mencionado, portanto atenderam a norma. Conforme a equação estimada para a IE24h, pode-se substituir as partículas de madeira por partículas de resíduo UKP em $41 \%$, mantendo os painéis dentro dos valores máximos estipulados pela norma mencionada .

A Figura 6 apresenta a relação entre a porcentagem de resíduo UKP e a taxa de não retorno em espessura (TNRE) dos painéis aglomerados, sendo que a relação se mostrou significativa para a regressão quadrática. Assim como no inchamento em espessura, a TNRE aumentou com o aumento da quantidade de resíduo utilizado, fato que comprova a associação direta entre tais propriedades.

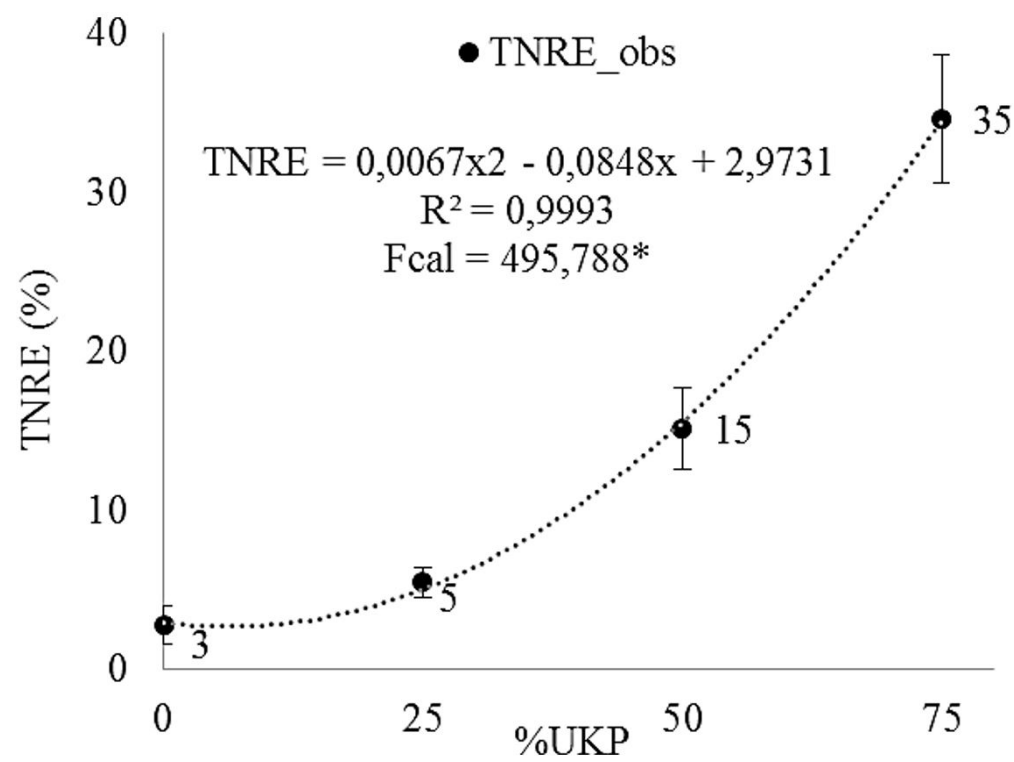

Figura 6. Taxa de não retorno em espessura dos painéis MDP, onde $\left(^{*}\right)$ - significativo ao nível de $5 \%$ de significância. Figure 6. Non-return rate in thickness of MDP panels, where $(*)-$ significant at the $5 \%$ level of significance.

A TNRE, que mede o percentual de inchamento residual no painel, demonstra que quanto maior o percentual de UKP na composição do miolo, maior a dificuldade de retorno a espessura inicial do painel antes da imersão em água.

De modo geral, o acréscimo do resíduo UKP nos painéis prejudicou as propriedades físicas. Este resultado pode ser explicado pelo gradativo aumento da razão de compactação (Figura 2), promovendo a diminuição da quantidade de adesivo por unidade de área das partículas, os painéis produzidos possuem maior quantidade de material lignocelulósico, possuindo assim, mais sítios ativos para a molécula de água se ligar. Usualmente painéis com maiores razões de compactação resultam em valores maiores de absorção de água e inchamento em espessura ambos após 24 horas de imersão em água, prejudicando a estabilidade dimensional dos painéis (IWAKIRI et al., 2004). Como as fibras são provenientes de um processo químico de polpação, além de já terem passado por um processo que "altera" a sua superfície, ainda existem os resíduos químicos que podem interferir na ligação adesiva. Outro fator que pode ter afetado as propriedades físicas dos painéis foi a composição química do material utilizado. O resíduo UKP e madeira de eucalipto utilizados nesse trabalho foram avaliados anteriormente por César (2015), que observou teores de lignina total e extrativos de 15,5 e 16\%, respectivamente e para a madeira de Eucalyptus sp. de 14,2 para os extrativos e 27,5\% para lignina total. Observa-se, pelos dados obtidos, que o resíduo apresenta menor quantidade de lignina e maior quantidade de extrativos. Tal fato era esperado, uma vez que o processo de cozimento da indústria de celulose visa a remoção da lignina, o que deixou pequena quantidade no resíduo e possivelmente parte do licor presente no resíduo possa ter sido quantificada como extrativo pela metodologia utilizada pela autora. De acordo com Silva et al. (2015), essas variações podem interferir na cura do adesivo, bem como, na estabilidade dimensional dos painéis.

Em alguns casos, em que as variações quantitativas e os tipos de extrativos representam importante parte no processo produtivo, podem ocorrer problemas na sua taxa de cura. Com isso, podem ser 
observadas baixas resistências à umidade em produtos acabados. Problemas desses tipos devem ser avaliados quando forem utilizadas espécies que possuam extrativos que afetem negativamente o processo de composição do painel (MALONEY, 1993). Espécies com baixo teor de extrativos são as mais indicadas para a produção de aglomerado convencional (CLOUTIER, 1998). Em contrapartida, a presença de importantes grupos funcionais como hidroxil fenólico, hidroxil alifático, carbonil, éter alquilarílico, bifenil, éter diarílico, fenilpropano, guaiacil e siringil (HEISS-BLANQUET et al., 2011; YAN et al., 2017) na lignina favorece a adesão com outros grupos funcionais do aderente. É importante ressaltar que não foi utilizado nenhum tipo de parafina ou cera, para diminuir o caráter hidrofílico dos materiais lignocelulósicos e nenhum tratamento no resíduo como lavagem ou adição de produto químico.

A Figura 7 apresenta a relação entre a porcentagem de resíduo UKP e a propriedade tração perpendicular (TP) dos painéis MDP. A regressão linear foi a que mais se adequou aos dados, sendo essa estatisticamente significativa, com a redução dos valores médios à medida que se aumentou a quantidade de resíduo.

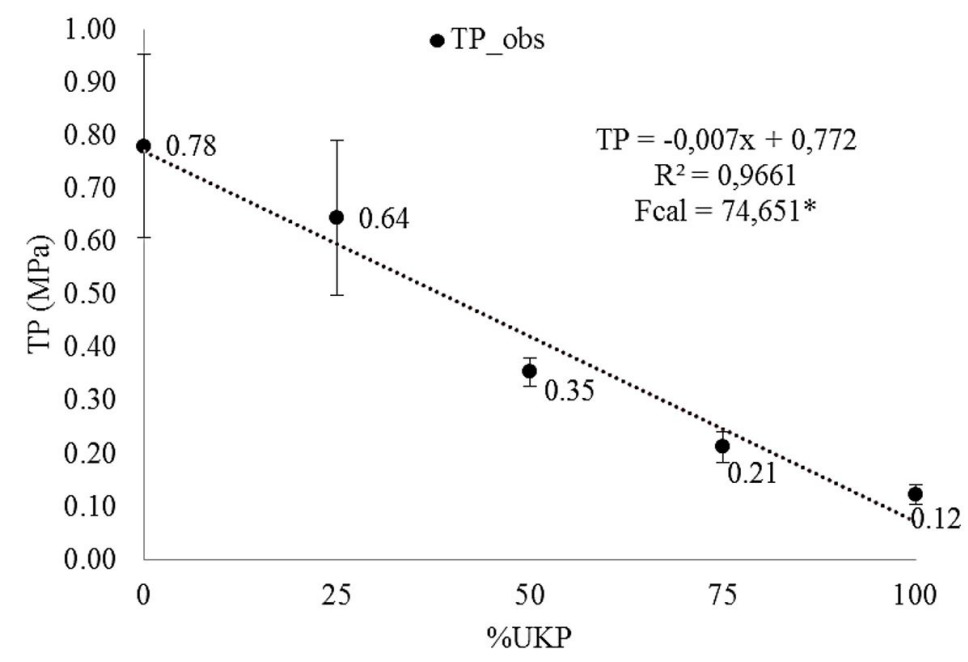

Figura 7. Tração perpendicular dos painéis MDP, onde $\left(^{*}\right)$ - significativo ao nível de $5 \%$ de significância.

Figure 7. Perpendicular traction of MDP panels, where $(*)-$ significant at the $5 \%$ level of significance.

A tendência de decréscimo dos valores médios ocorreu em parte pela diminuição da quantidade de adesivo por partícula devido à baixa densidade do resíduo utilizado $\left(0,325 \mathrm{~g} / \mathrm{cm}^{3}\right)$, consequentemente ocasionando maior volume (IWAKIRI et al., 2004). A baixa quantidade de lignina presente no resíduo quando comparada a madeira de eucalipto também contribui com a baixa resistência mecânica dos MDP, devido as suas características intrínsecas de aumentar a rigidez da parede celular e contribuir na consolidação das partículas em função de funcionar como um adesivo natural (SILVA et al., 2015).

A diminuição da granulometria do resíduo em trabalhos futuros pode facilitar e melhorar a homogeneização das partículas de madeira e resíduo, que por sua vez pode melhorar a dispersão do adesivo e a colagem, é importante ressaltar que o encolamento foi realizado de forma manual. Porém, conforme caracterização granulométrica do resíduo realizada por César (2015), a quantidade de material retido nas peneiras com 40 mesh ou mais é, praticamente insignificante, justificando o não peneiramento do resíduo para a etapa de fabricação dos painéis neste estudo.

Essa tendência de decréscimo também foi observada por Mendes et al. (2010), quando se avaliou os valores de tração perpendicular em função do aumento das porcentagens de casca de café (25, 50 e 75\%), em painéis aglomerados de Eucalyptus urophylla. Os valores de tração perpendicular decresceram de $0,30 \mathrm{MPa}$ a 0,15 MPa, conforme se aumentava a porcentagem de casca de café. Scatolino et al. (2013) observaram valores de tração perpendicular de 1,1 MPa para painéis produzidos exclusivamente com madeira de Pinus oocarpa, valor que foi consideravelmente superior aos encontrados neste trabalho para todas as porcentagens de UKP.

A relação linear entre o MOR e a adição do resíduo UKP mostrou-se significativa, assim como observado para MOE. Houve um decréscimo dos valores de MOE e MOR com o aumento da porcentagem de resíduo UKP no miolo dos painéis (Figura 8 e 9). 
Andrade et al. - Inclusão do resíduo de polpação da celulose na produção de painéis aglomerados de média densidade

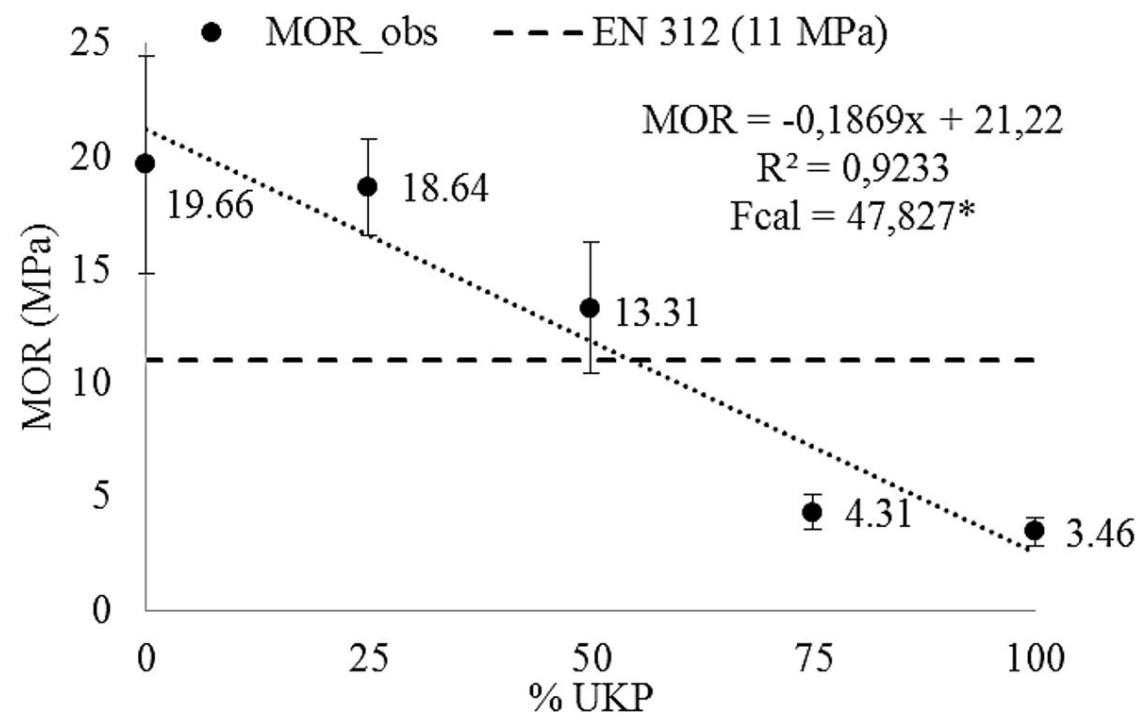

Figura 8. Módulo de ruptura dos painéis MDP, onde $(*)$ - significativo ao nível de $5 \%$ de significância.

Figure 8. Modulus of rupture of MDP panels, where $(*)$ - significant at the $5 \%$ level of significance.

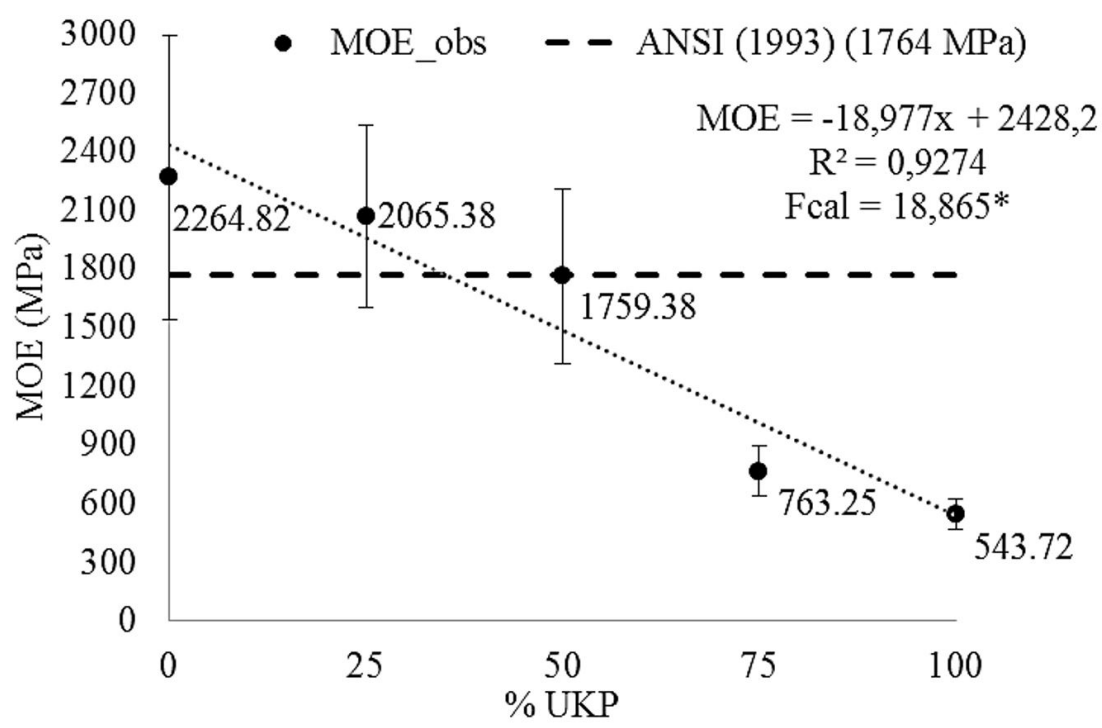

Figura 9. Módulo de elasticidade dos painéis MDP, onde $\left(^{*}\right)$ - significativo ao nível de $5 \%$ de significância.

Figure 9. Modulus of elasticity of MDP panels, where $\left(^{*}\right)$ - significant at the $5 \%$ level of significance.

Melo et al. (2009), investigaram a associação de casca de arroz com a madeira de Eucalyptus grandis em painéis aglomerados e Scatolino et al. (2013), avaliando diferentes percentagens de sabugo de milho em associação com madeira de Pinus oocarpa, observaram uma diminuição dos valores das propriedades mecânicas MOE, MOR e TP em função do aumento da porcentagem de resíduo nos painéis. Os autores justificaram a tendência decrescente pela baixa densidade básica do resíduo, diminuindo a disponibilidade de adesivo por partícula.

A norma ANSI (American National Standard Institute) (ANSI, 1993) estipula o valor mínimo para MOR de 11 MPa para painéis produzidos com uréia-formaldeído. Diante disso, em comparação com a norma, os painéis produzidos com até 50\% de UKP atingiram o valor mínimo exigido. A mesma norma estipula valores mínimos para o MOE de $1764 \mathrm{MPa}$ para painéis aglomerados produzidos com adesivo uréia-formaldeído, sendo assim, em comparação com essa norma, os painéis produzidos com $25 \%$ de UKP atingiram o valor mínimo estipulado. Conforme a equação estimada para a MOE e MOR, pode-se substituir as partículas de madeira por partículas de resíduo UKP em 35 e 55\%, respectivamente, mantendo os painéis dentro dos valores mínimos estipulados pelas respectivas normas mencionadas. 


\section{CONCLUSÃO}

Para as todas as propriedades avaliadas de AA2H, AA24H, IE2H, IE24H e TNRE, foi observado um aumento dos valores dessas propriedades à medida que aumentou a porcentagem de resíduo UKP. As propriedades mecânicas MOE, MOR e TP dos painéis produzidos diminuíram à medida que a porcentagem de resíduo aumentava. Recomenda-se a utilização de até 35\% de substituição de partículas de madeira por partículas do resíduo UKP para produção dos painéis MDP, permitindo, assim, uma destinação do rejeito na geração de novos produtos, mantendo as propriedades físicas e mecânicas dentro dos valores exigidos pelas normas. De maneira geral, o acréscimo do resíduo UKP para produção de painéis MDP, ocasionou decréscimo nas propriedades mecânicas e aumento das propriedades físicas. Diante dos resultados observados, novos estudos são necessários, com intuito de adequar o processo produtivo, como variação de granulometria e novos tratamentos químicos buscando viabilizar o uso deste resíduo em maiores quantidades na produção de painéis MDP.

\section{AGRADECIMENTOS}

Os autores agradecem a Fundação de Amparo à Pesquisa do Estado de Minas Gerais_-FAPEMIG, Coordenação de Aperfeiçoamento de Pessoa de Nível Superior-CAPES, ao Conselho Nacional de Desenvolvimento Científico e Tecnológico-CNPq e a Rede Brasileira de Pesquisa em Compósitos e Nanocompósitos Lignocelulósicos-RELIGAR.

\section{REFERÊNCIAS BIBLIOGRÁFICAS}

ANSI - AMERICAN NATIONAL STANDARD INSTITUTE. ANSI A208.1: Mat-formed wood particleboard: Specification. Gaithersburg, 1993.

ABNT - ASSOCIAÇÃO BRASILEIRA DE NORMAS TÉCNICAS. ABNT NBR 14810: chapas de madeira aglomerada parte 3: métodos de ensaio. Rio de Janeiro, 2006. 51 p.

ABNT - ASSOCIAÇÃO BRASILEIRA DE NORMAS TÉCNICAS. ABNT NBR 11941: madeira: determinação da densidade básica. Rio de Janeiro, 2003. 6 p.

ASTM - AMERICAN SOCIETY FOR TESTING AND MATERIALS. ASTM D 1037: standard methods of evaluating properties of wood-base fiber and particles materials. Philladelphia, 2006.

BARROS FILHO, R. M. Painéis Aglomerados a Base de Bagaço de Cana-de-Açúcar e Resinas Uréia Formaldeído e Melanina Formaldeído. 2009. 99 p. Dissertação (Mestrado em Engenharia de Materiais da Rede Temática em Engenharia de Materiais - REDEMAT - UFOP, CETEC, UEMG). Universidade Federal de Ouro Preto, Ouro Preto, 2009.

CÉSAR, A. A. S. Tratamento do resíduo da depuração (UKP) da indústria de celulose para produção de chapas minerais. 2015. 103 p. Tese (Doutorado em Ciência e Tecnologia da Madeira) - Universidade Federal de Lavras, Lavras, 2015.

CLOUTIER, A. Oriented stranboard (osb): raw material, manufacturing process, properties of wood-base fiber and particle materials. In: INTERNATIONAL SEMINAR ON SOLID WOOD PRODUCTS OF HIGH TECHNOLOGY, 1., 1998, Belo Horizonte. Anais... Belo Horizonte: SIF, 1998. p. 173-185.

DIN - DEUTSCHES INSTITUT FUR NORMUNG. DIN 52362: Testing of wood chipboards, bending test, determination of bending strength. Berlin, 1982. $40 \mathrm{p}$.

EN - EUROPEAN COMMITTEE FOR STANDARDIZATION. EN 312: Particleboard - Specifications. Bruxelas, 2003.

EN - EUROPEAN COMMITTEE FOR STANDARDIZATION. EN 323. Wood based panels -Determination of density. Bruxelas: APA Europe: 1993.

GAMA, R. O.; DIAS, F. M; NASCIMENTO, M. F.; LAHR, F. R. Painéis de partículas de rejeito UKP/BKP da indústria de celulose e de resíduos de Pinus elliottii: resistência ao arrancamento de parafusos e resistência à tração perpendicular às faces. Construindo, Belo Horizonte, v. 2, n. 1, p. 35-39, 2010. 
Andrade et al. - Inclusão do resíduo de polpação da celulose na produção de painéis aglomerados de média densidade

GULER, C.; BUYUKSARI, U. Effect of production parameters on the physical and mechanical properties of particleboards made from peanut (Arachis hypogaea L.) husk. BioResources, Raleigh, v. 6, n.4, p.5027-5036, 2011.

HEISS-BLANQUET, S.; ZHENG, D.; FERREIRA, N. L. Effect of pretreatment and enzymatic hydrolysis of wheat straw on cell wall composition, hydrophobicity and cellulose adsorption. Bioresource Technology, Lucknow, v. 102, n. 10, p. 5938-5946, 2011.

IBÁ - INDÚSTRIA BRASILEIRA DE ÁRVORES Relatório anual de 2016. São Paulo: IBÁ, 2017.

IWAKIRI, S.; SHIMIZU, J.; SILVA, J. C.; DEL MENEZZI, H. S.; PUEHRINGHER, C. A. VENSONS, I. LARROCA, C. Particle board manufacturing from Grevillea robusta A. Cunn. ExR. Br. Revista Árvore, Viçosa, v. 28, n. 6, p. 883-887, 2004.

IWAKIRI, S.; CUNHA, A. B.; ALBUQUERQUE, C. E. C.; GORNIAK, E.; MENDES, L. M. Resíduos de serrarias na produção de painéis de madeira aglomerada de eucalipto. Scientia Agraria, Piracicaba, v.1, n. 1/2, p. 23$28,2000$.

MALONEY, T. M. Modern particleboard and dry process fiberboard manufacturing. 2.ed. São Francisco: M. Freeman, 1993. 689 p.

MELO, R. R.; SANTINIM E. J.; HASELEIN, C. R.; STARGELIN, D. M. Propriedades físico-mecânicas de painéis aglomerados produzidos com diferentes proporções de madeira e casca de arroz. Ciência Florestal, Santa Maria, v. 19, n. 3-4, p. 449-460, 2009.

MENDES, R. F.; MENDES, L. M.; GUIMARÃES JÚNIOR, J. B.; SANTOS, R. C.; CÉSAR, A. A. S. Association effect of sugar cane bagasse, type and levels of adhesive on particleboard production. Ciência Florestal, Santa Maria, v. 22, n.1, p.161-170, 2012.

MENDES, R. F.; MENDES, L. M.; GUIMARÃES JÚNIOR, J. B.; MORI, F. A.; CÉSAR, A. A. S. Effect of the incorporation of coffee husks on the physico-mechanical properties of Eucalyptus urophylla S. T. Blake particleboards. Ciência e Agrotecnologia, Lavras, v. 34, n. 3, p. 610-617, 2010.

MESQUITA, R. G. de. A. et al. Inclusão de feixes de sisal na produção de painéis MDP de eucalipto. Scientia Forestalis, Piracicaba, v. 43, n.105, p.75-82, 2015.

MOHR, B.J.; NANKO, H.. KURTIS, K. E. Durability of kraft pulp fiber-cement composites to wet/dry cycling. Cement \& Concrete Composites, Vancouver, v. 27, n. 4, p. 435-448, 2005.

NEUBERGER, R. Boas Práticas de Operação para Redução de Odores na Produção de Celulose Kraft: Uma Abordagem Qualitativa. 2008. 125 p. Dissertação (Mestrado em Engenharia de Processos Químicos e Bioquímicos) - Universidade de Mauá, São Caetano do Sul, 2008.

PEDRAZZI, C.; HASELIN, C. R.; SANTINI, EL. J.; SCHNNEIDER, P. R. Qualidade de chapas de partículas de madeira aglomerada fabricadas com resíduos de uma indústria de celulose. Ciência Florestal, Santa Maria, v. 16, n. 2, p. 201-212, 2006.

PIERRE, F.C.; BALLARIN, A. W.; HERNANDO, L. P et al. Caracterização física de painéis aglomerados de Eucalyptus grandis com adição de resíduos industriais madeireiros. Cerne, Lavras, v. 20, n. 2, p. 321-328, 2014

SCATOLINO, M. V.; PROTASIO, T. P.; MENDES, R. F.; MENDES, L. M. Thermal stability of Pinus oocarpa and maize cob particleboards. Ciência e Agrotecnologia, Lavras, v. 39, n. 4, p.348-354, 2015.

SCATOLINO, M. V.; SILVA, D. W.; MENDES, R. F.; MENDES, L. M. Use of cob for production of particleboard. Ciência e Agrotecnologia, Lavras, v. 37, n. 4, p. 330-337, 2013.

SEKALUVU, L.; TUMUTEGYEREIZE, P. KIGGUNDU, N. Investigation of factors affecting the production and properties of maize cob-particleboards. Waste and Biomass Valorization, Albi, v.5, n. 1. p.27-32, 2014.

SILVA, D. W.; COSTA, A. M.; GUIMARÃES JÚNIOR, J. B.; PROTÁSIO, T. P.; MENDES, R. F.; MENDES, L. M. Addition of different proportions of castor husk and pine wood in particleboards. Waste and Biomass Valorization, Albi, v. 7, n. 34, p.1-7, 2016.

SILVA, D. W.; FARRAPO, C. L.; RIBEIRO, D. P.; MENDES, R. T.; MENDES, L. M.; SCOLFORO, J. R. S. MDP com partículas de eucalipto e palha de milho. Scientia Forestalis, Piracicaba, v. 43, n.108, p.853-862, 2015. 
SORATTO, D.; CUNHA, A. B.; VITAL, B.; CARNEIRO, A. C.O.; COSTA, F. R. Efeitos da adição de cavaco com casca na qualidade de painéis MDP produzidos com Eucalyptus sp. Ciência da Madeira, Pelotas, v. 4, n. 1, p. 46-59, 2013.

TONOLI, G.H.D.; SANTOS, S. F.; TEIXEIRA, R. S.; SILVA, M. A. P.; LAHR, F. R.; SILVA, F. H. P.; SAVASTANO JUNIOR, H. Effects of eucalyptus pulp refining on the performance and durability of fibre-cement composites. Journal of Tropical Forest Science, Kepong, v. 25, n.3, p. 400-409, 2013.

YAN, L.; GOU, G.; CUI, Y.; WANG, Q. Liquefaction of lignin in hot-compressed water to phenolic feedstock for the synthesis of phenol-formaldehyde resins. Composites Part B: Engineering, In press, New Orleans, v. 112, p. 8-14. 2017.

YENIOCAK, M.; GOKTAS, O.; ERDIL, Y. Z.; MEHMET, A. Investigating the use of vine pruning stalks (Vitis Vinifera L. CV. Sultani) as raw material for particleboard manufacturing. Wood Research, Bratislava, v. 59, n. 1, p.167-176, 2014.

Recebido em: 18/10/2017

Aceito em: 14/05/2018 